\title{
Routing and Spectrum Assignment Integrating Machine-Learning-Based QoT Estimation in Elastic Optical Networks
}

\author{
Matteo Salani ${ }^{1}$, Cristina Rottondi ${ }^{2}$ and Massimo Tornatore ${ }^{3}$
}

\begin{abstract}
Machine Learning (ML) is under intense investigation in optical networks as it promises to lead to automation of a variety of management tasks, as amplifier gain equalization, fault recognition, Quality of Transmission (QoT) estimation, and many others. Though several studies focus on each of these specific tasks, the integration of ML-based estimations inside Routing and Spectrum Assignment (RSA) is still largely unexplored.

This paper moves towards such integration. We develop a framework that leverages the probabilistic outputs of a MLbased QoT estimator to define the reach constraints in an Integer Linear Programming (ILP) formulation for RSA in an elastic optical network. In this integrated procedure, the RSA problem is solved iteratively by updating the reach constraints based on the outcome of a QoT estimator, to exclude lightpaths with unacceptable QoT. In our numerical evaluation, the proposed integrated method achieves savings in spectrum occupation up to $30 \%$ (around $20 \%$ on average) compared to traditional ILPbased RSA approaches with reach constraints based on margined analytical models.
\end{abstract}

Index Terms-Machine Learning; Routing and Spectrum Assignment; QoT estimation;

\section{INTRODUCTION}

Design and management of optical networks is continuously evolving to cope with an Internet traffic that is still growing at $40 \%$ annually [1]. In the latest generation of Elastic Optical Networks (EONs), coherent optical transmission [2] has been adopted to deploy highly spectrally-efficient and tunable modulation formats. Additionally, new flex-grid technologies [3] that subdivide fiber spectrum in fine-grained slots have been introduced. Such technologies enabled significant reductions of spectrum occupation with respect to traditional fixed-grid networks [4], but they also multiplied the number of tunable design parameters, thus largely increasing the number of possible network configurations and making network management much more challenging.

The main problem in EON management is the so-called Routing and Spectrum Assignment (RSA), which consists in assigning to every traffic request a lightpath (i.e, an all-optical end-to-end circuit) connecting a source to a destination node over a commensurate spectrum portion. To obtain a feasible

\footnotetext{
${ }^{1} \mathrm{M}$. Salani is with the Dalle Molle Institute for Artificial Intelligence, University of Lugano (USI), University of Applied Science and Arts of Southern Switzerland (SUPSI) - Switzerland matteo.salani@ supsi.ch

${ }^{2} \mathrm{C}$. Rottondi is with the Dept. of Electronics and Telecommunications, Politecnico di Torino, Italy cristina.rottondiapolito.it

${ }^{3} \mathrm{M}$. Tornatore is with the Dept. of Electronics, Information anad Bioengineering, Politecnico di Milano, Italy massimo.tornatore@polimi.it
}

EON RSA solution, the Quality of Transmission (QoT) of each lightpath must be estimated before deployment to verify which modulation formats can be safely used, considering that physical-layer impairments can affect signal propagation. Hence, QoT estimation is an essential preliminary step for achieving effective RSA. Existing techniques for QoT estimation mostly rely on approximated analytical models (as the Gaussian-noise model [5]) that, to provide an accurate estimation, require perfect knowledge of transmission parameters such as the type of used fiber, the noise figure of amplifiers, etc. In a realistic network deployment, these parameters are not always perfectly known (e.g., due to an incomplete equipment inventory, or in the case of an alien wavelengths [6]). Due to such inaccuracies, standard approximated models might lead to significant underutilization of network resources ${ }^{1}$. Other more precise estimation approaches (as those based on the Split Step Fourier Transform [8]) are computationally too demanding to allow for scalable and real-time decision making.

Machine Learning (ML) is regarded as a potentially disruptive methodology to overcome the shortcomings of existing techniques. ML promises to return fast and accurate QoT estimation by leveraging the knowledge extracted from field data (e.g., the pre-FEC Bit Error Rate (BER), or the Optical Signal to Noise Ratio (OSNR) of existing lightpaths) acquired by means of Optical Performance Monitors (OPMs) [9] deployed in strategic points of the network (e.g., at receiving nodes). Several works exist in the field of QoT estimation and RSA (a summary is reported in the next section). However, up to now, existing research has addressed RSA and QoT estimation separately. The integration of ML-based QoT estimation inside an RSA framework for EONs is still in its infancy. This paper moves in this direction by proposing a framework that integrates the probabilistic output of a ML classifier for QoT estimation [10] in an Integer Linear Program (ILP) formulation for RSA, considering multiple modulation formats. Once an initial solution is found, the classifier can be queried iteratively to further refine it by including features about the neighbor channels of each lightpath, to account for inter-channel interference. This allows to insert additional constraints in the ILP formulation to prevent that the deployment of neighboring channels causes excessive QoT degradation at the receiver nodes. We show that, in our case study, the proposed frame-

\footnotetext{
${ }^{1}$ Ref. [7] reports that throughput gains from $25 \%$ up to $300 \%$ - depending on the network topology - can be achieved by avoiding margins traditionally introduced to account for the uncertainty of physical-layer parameters.
} 
work saves up to almost $30 \%$ in spectrum occupation with respect to a traditional RSA solution with margined reach computations.

The remainder of the paper is organized as follows. Section II briefly overviews the related scientific literature, whereas some background notions are provided in Section III. Section IV describes our proposed network-planning framework. The ILP model that solves the Routing, Modulation format and Spectrum Assignment (RMSA) problem and the integration of ILP formulation and QoT classifier are described in Section V and assessed in Section VI. Concluding remarks are provided in the last Section.

\section{RELATED WORK}

\section{A. Quality-of-Transmission Estimation}

Several studies on lightpath QoT prediction have recently appeared: Ref. [11] adopts a cognitive Case Based Reasoning (CBR) approach, which stores in a database a list of Qfactor measurements, together with a set of characteristics of the associated lightpaths. When a new lightpath has to be deployed, the table entries which exhibit highest similarity to the candidate lightpath are used to make an estimation of the expected Q-factor. A similar approach is adopted in [12] to tune design margins in presence of unknown network parameters: a controller collects and stores field data, which are ingested by a prediction tool that outputs an OSNR estimation based on educated guesses on the unknown network parameters. In [13], Gaussian-Processes nonlinear Regression is adopted to predict the BER of an optical communication system using as features the channel input power, lightpath length, symbol rate and inter-channel spacing. Two alternative ML approaches named network kriging and norm $\mathcal{L}_{2}$ minimization are applied in [14]-[17] to perform QoT estimation. These methods require the installation of probe lightpaths carrying dummy traffic to acquire field measurements to compute an estimation of the Q-factor of already established or candidate lightpaths. In [10], Random Forests are used to predict whether the BER of unestablished lightpaths will exceed a given threshold, based on set of features representing the transmission parameters of the lightpath. In this paper, we will leverage this approach (see Section III for further details), as it does not require the installation of probe lightpaths and considers several modulation formats.

\section{B. Integration of QoT Predictors and RSA Techniques}

Though several models for RSA in EONs have been investigated in the past decade (see [18] for a recent survey), only a few preliminary attempts to integrate the outputs of QoT estimators in traditional RSA have appeared. In [19], the output of a neural-network estimator is exploited by a heuristic algorithm for dynamic routing and spectrum assignment in a multicast scenario. The authors of [20] propose a ML-based approach for inter-core crosstalk estimation in optical networks with multicore fibers. The proposed regressor is queried by a heuristic algorithm for core, route and spectrum assignment. In [21], a ML-based traffic predictor and a QoT estimator leveraging deep neural networks are integrated in a Routing, Modulation format and Spectrum Assignment (RMSA) heuristic algorithm. Differently, in this study we focus on a static scenario and assume the availability of multiple modulation formats. The QoT estimator is called iteratively by an ILP formulation for RMSA, whose constraints gradually gain knowledge on neighbor channels, based on the optimal network configuration calculated at the previous round.

\section{BACKGROUND}

\section{A. QoT ML Classifier}

A random-forest binary classifier is adopted in [10] to predict the probability that the BER of unestablished lightpaths will exceed a given threshold (for additional details about the choice of the learning algorithm and for a thorough performance assessment of the classifier, the reader is referred to [10]). As depicted in Fig. 1, the classifier is trained with a dataset of input instances consisting in a list of features that characterize a lightpath: more in detail, an instance relative to a given lightpath $k$ includes as features the lightpath total length $\mathcal{L}_{k}$ and maximum link length $\mathcal{L}_{k}^{\max }$, the number of traversed links $l_{k}$, the amount of traffic $d$ to be transmitted and the modulation format $m$ to be adopted for transmission. Optionally, the classifier may consider additional features characterizing the neighbor channels of lightpath $k$ (represented in the grey dashed portions of Fig. 1): such additional features include guardband size $g_{l}, g_{r}$, traffic volume $d_{l}, d_{r}$ and modulation format $m_{l}, m_{r}$ of the spectrally nearest left and right channels (co-propagating with the considered lightpath along at least one of its links).

Given a traffic request, alternative configurations of routes and modulation formats are given as input to the classifier and, for each of them, the classifier outputs a probability $p_{t h}$ that the ligthpath configuration will satisfy a given threshold $t h$ on the BER measured at the receiver. Then, to obtain a yes/no answer, the probability output is binarized according to the following rule: given a reference value $p_{t h}^{*}$, the ligthpath is considered to be feasible if $p_{t h} \geq p_{t h}^{*}$, infeasible otherwise. Typically, $p_{t h}^{*}$ is set to 0.5 . Note that higher (resp. lower) values of $p_{t h}^{*}$ will lead to less false-positive occurrences ${ }^{2}$ (resp. false negatives). Note also that the inclusion of features related to neighbor co-propagating channels improves the classification accuracy for lightpaths with BER values closely approaching th.

\section{B. Qtool for Framework Validation}

Due to the unavailability of lightpaths' BER measurements gathered from real networks, these values are generated using the Qtool described in [10]. The Qtool generates realistic instances of the uncoded BER at the input of the FEC soft decoder (pre-FEC BER) by simulating a linear optical communication system affected by chromatic dispersion and additive white Gaussian noise (AWGN). Under the AWGN assumption,

\footnotetext{
${ }^{2}$ We denote as false positives the above-threshold lightpaths that are erroneously classified as feasible, whereas we denote as false negatives the below-threshold lightpaths that are incorrectly classified as infeasible.
} 


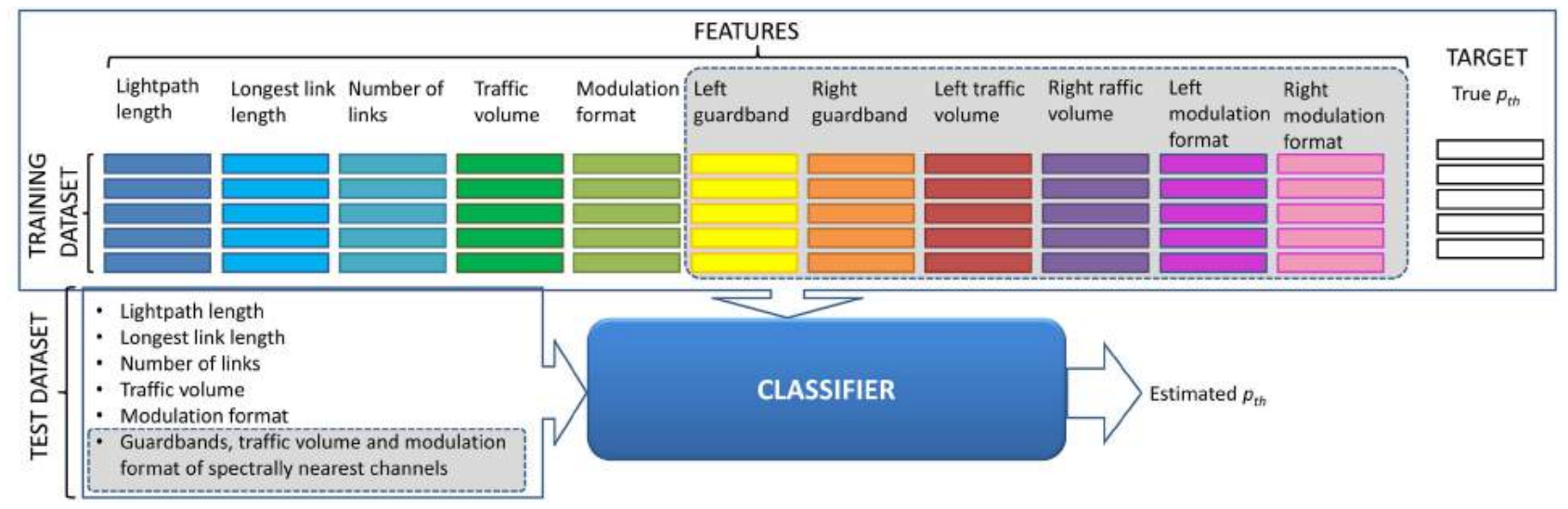

Fig. 1: The classification method for QoT estimation adopted in [10]. Left and right attributes are referred to the spectrally nearest neighbor channel co-propagating with the lightpath on at least one link.

a)

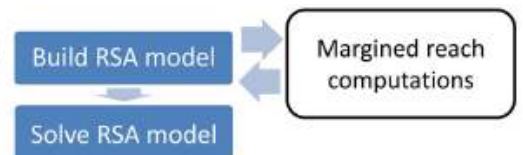

b)

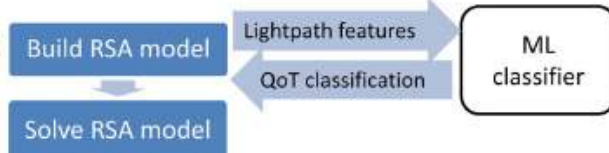

Solve RSA model

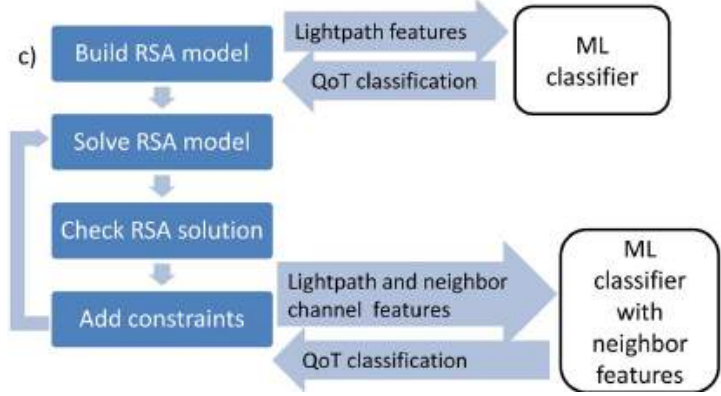

Fig. 2: Margined (a), ML-based (b) and iterative (c) RMSA frameworks.

the pre-FEC BER depends on the pre-FEC signal-to-noise ratio (SNR) and on the modulation format. For a given lightpath, the pre-FEC SNR can be estimated by a link budget, taking into account the transmitted power, gains and losses along the route. More in detail, the Qtool considers transparent links of dispersion uncompensated standard single-mode fibers where the signal power is restored by identical optical amplifiers equally spaced over the links $(100 \mathrm{~km})$, with gain $20 \mathrm{~dB}$ and noise figure $5 \mathrm{~dB}$. On top of this link budget, we add a negative-exponential random additonal penalty, mimicking uncertainity in transmission parameter knowledge. Following [5], in the remainder of the paper we will set the pre-FEC BER threshold as $t h=4 \cdot 10^{-3}$.

\section{Integrated Network PlanNing Framework}

The RSA problem can be summarized as follows: given a network topology and set of traffic demands between source- destination node pairs, for each demand a lightpath must be selected to route the traffic from source to destination and an optical channel must be assigned to the lightpath to accommodate the demand in a commensurate spectrum portion. Spectrally adjacent channels must be interleaved by guardbands to ensure proper signal filtering and switching. The number of transceivers to be allocated in each channel depends on the traffic volume to be served and on the spectral efficiency of the modulation format used for transmission. If multiple modulation formats can be adopted, the problem evolves in RMSA. In such case, the problem must include reach constraints that define the feasibility of the deployment of a ligthpath along a given route ${ }^{3}$.

\section{A. Baseline Design Approach with Margined Reach Con- straints}

In recent literature, reach constraints are set based on pre-computed reach values obtained via margined analytical formulas (see Fig. 2a). Marginations are necessary to account for uncertainties in physical layer characterization (e.g., amplifier noise figures, switching equipment and fiber propagation losses) and in the actual network configuration (e.g., interchannel crosstalk due to neighbor lightpaths). Such formulas ensure an acceptable QoT, but they are often too conservative and may lead to underutilization of network resources. In the remainder of the paper, this design approach will be named as margined.

\section{B. ML-Based Reach Constraint Design Approach}

As depicted in Fig. 2b, a first intuitive integration of RMSA with ML consists in replacing the margined reach computations with a ML-based QoT classifier that, on input of a query for each possible combination of traffic volume, route and modulation format, returns the probability that the considered combination is feasible. Based on such output, reach constraints can then be defined and integrated in the RMSA model. As the classifier is trained with a consistent

\footnotetext{
${ }^{3}$ Note that higher traffic means a larger number of transceivers/signals in the same optical channel, hence higher intra-channel interference.
} 
amount of monitoring data gathered from already activated lightpaths, it is expected to capture the actual physical-layer characteristics more accurately than margined formulas. In the following, this approach will be referred to as ML-based.

\section{Iterative Reach Constraint Design Approach}

A limitation of ML-based QoT estimators is that they are prone to errors (i.e., a lightpath could be erroneously classified as feasible, despite its actual received BER exceeds the threshold). Such errors could be reduced, if information on the spectrally adjacent channels co-propagating with the considered lightpath are provided as additional input to the classifier (including, e.g., their traffic volume, modulation format and guardband spacing). Unfortunately, even considering only the spectrally nearest left and right neighbor channels, an a-priori ML-based estimation for all possible configurations of a lightpath and its neighbor channels is computationally infeasible in realistic scenarios. As an example, let us assume that traffic demands may have 10 different sizes (e.g., from 50 Gbps to 400 Gbps with 50 Gbps granularity), that 6 modulation formats are available and that guardbands may have 10 different widths, for each ligthpath the feasibility of $6^{3} \cdot 8^{3} \cdot 10^{2}=11,059,200$ configurations should be precomputed. Therefore, integrating information on neighboring channels in RSA models introduces huge scalability issues ${ }^{4}$.

To overcome such limitations, we propose an iterative procedure (see Fig. 2c) that, after finding a solution to the initial RMSA formulation, for each lightpath in the solution, issues a query to the ML classifier containing the exact features of that lightpath and of its neighboring channels (hence, there is no need to account for all possible cases as in the previous example). If the classifier returns a negative outcome (i.e., the ligthpath QoT is not acceptable), an additional constraint is added that excludes from the RMSA solution the unacceptable deployment of the ligthpath and its neighbors. The process is repeated iteratively, until either a feasible solution for all lightpaths is found, or a maximum number of iterations is reached. In the following sections, this approach will be referred to as iterative.

\section{The RMSA Problem Formulation}

\section{A. Assumptions and Notations}

We consider an arbitrary network topology represented by an undirected graph $\mathcal{G}=(\mathcal{V}, \mathcal{E})$, where $\mathcal{V}$ is the set of nodes and $\mathcal{E}$ is the set of bidirectional links. Each link $e \in \mathcal{E}$ is characterized by a length $L_{e}$. A static traffic matrix $D=\left[d_{s d}\right]$ of traffic requests between each node pair $(s, d) \in T=\{\mathcal{V} \times \mathcal{V}: s \neq d\}$ defines the traffic volume generated by source node $s$ and directed to destination node $d$. To simplify the notation, in the following a traffic request between the $(s, d)$ pair will be denoted as an element $t$ of set $T$. For each request $t$, a set of candidate lightpaths $K_{t}$ is predefined. We assume that such set contains the $\bar{k}$ shortest

\footnotetext{
${ }^{4}$ In our considered scenario, the overall number of evaluations considering an all-to-all traffic matrix in a network with 14 nodes and 22 links would be in the order of $10^{9}$.
}

paths (i.e., $\left|K_{t}\right|=\bar{k}$ ). The length of a lightpath is defined as $\mathcal{L}_{k}=\sum_{e \in k} L_{e}$. For each lightpath, the length of its longest link is denoted as $\mathcal{L}_{k}^{\max }=\max _{e \in k} L_{e}$. The number of links traversed by lightpath $k$ is denoted as $l_{k}=|\{e \in \mathcal{E}: e \in k\}|$. We assume that optical fiber spectrum is subdivided in a flexible grid with standard slice width of $F \mathrm{GHz}$ and elastic transceivers operating at 28 Gbaud with optical bandwidth of $B \mathrm{GHz}$ (where $B$ is an integer multiple of $F$ ). Superchannels with multiple adjacent transceivers are used to serve traffic demands exceeding the capacity of a single transceiver. Spectrally neighboring (super)channels are separated by an optical guardband of $G \mathrm{GHz}$ (where $G$ is an integer multiple of $F$ ). Transceivers operate at one of the modulation formats within a given set $M$, which results in a capacity of $r_{m}$ Gbps. As we adopt a channel-based formulation, a set $C$ of candidate optical channels is precomputed. Each channel $c \in C$ occupies a predefined set of contiguous slices and is characterized by a bandwidth $w_{c}$.

\section{B. ILP Formulation}

Let us introduce the ILP formulation of the RMSA problem (adapted from [22] for a generic mesh topology).

Sets:

- $T$, set of $(s, d)$ pairs

- $K_{t}$, set of feasible lightpaths between $(s, d)$ pair $t \in T$

- $K=\cup_{t \in T} K_{t}$, set of all lightpaths

- $M$, set of modulation formats

- $C$, set of optical channels (group of contiguous spectrum slices)

\section{Parameters:}

- $Q$, positive constant (greater than the maximum number of transceivers that could be needed to serve a demand)

- $G$, guardband size

- $B$, bandwidth of a transceiver

- $\lambda_{k}^{m}, 1$ if the reach of modulation $m \in M$ exceeds the length of path $k \in K, 0$ otherwise

- $\phi_{k, k^{\prime}}, 1$ if paths $k \in K$ and $k^{\prime} \in K: k \neq k^{\prime}$, share at least one link, 0 otherwise

- $\psi_{c, c^{\prime}}, 1$ if channels $c \in C$ and $c^{\prime} \in C$ spectrally overlap, 0 otherwise

- $w_{c}$, bandwidth of channel $c \in C$

- $l_{k}$, length of path $k \in K$ in number of links

- $d_{t}$, traffic demand for $(s, d)$ pair $t \in T$

- $r_{m}$, capacity of a transceiver operating at modulation $m \in$ $M$

Variables:

- $b_{k}^{m}$, integer, number of transceiver pairs (one installed at node $s$, another at node $d$ ) with modulation $m \in M$ serving traffic $t \in T$ on path $k \in K_{t}{ }^{5}$

- $\beta_{k}^{m}$, binary, 1 if modulation $m \in M$ is used to serve traffic $t \in T$ on path $k \in K_{t}$

- $y_{k}^{c}$, binary, 1 if channel $c \in C$ is used along path $k \in K$

${ }^{5}$ Note that, as path $k$ is uniquely associated to a source-destination pair $t=(s, d)$, subscript $t$ is omitted to simplify the notation in all the variables. 


\section{Objective Function:}

$$
z=\min \alpha_{1} \cdot \sum_{k \in K} \sum_{m \in M} b_{k}^{m}+\alpha_{2} \cdot \sum_{c \in C} \sum_{k \in K} w_{c} \cdot l_{k} \cdot y_{k}^{c}
$$

Constraints:

$$
\begin{gathered}
\sum_{c \in C} \sum_{k \in K_{t}} y_{k}^{c}=1 \quad \forall t \in T \\
\sum_{m \in M} \sum_{k \in K_{t}} \beta_{k}^{m}=1 \quad \forall t \in T \\
\sum_{m \in M} \sum_{k \in K_{t}} r_{m} \cdot b_{k}^{m} \geq d_{t} \quad \forall t \in T \\
\beta_{k}^{m} \leq \lambda_{k}^{m} \cdot \sum_{c \in C} y_{k}^{c} \quad \forall m \in M, \forall k \in K \\
b_{k}^{m} \leq Q \cdot \beta_{k}^{m} \quad \forall m \in M, \forall k \in K \\
\sum_{m \in M} b_{k}^{m} \leq \sum_{c \in C}\left(w_{c}-G\right) \cdot y_{k}^{c} \quad \forall k \in K \\
y_{k}^{c}+y_{k^{\prime}}^{c^{\prime}} \leq 1 \\
\forall\left(c, c^{\prime}\right) \in C \times C \mid \psi_{c, c^{\prime}}=1 \\
\forall\left(k, k^{\prime}\right) \in K \times K \mid \phi_{k, k^{\prime}}=1
\end{gathered}
$$

The objective function (1) minimizes a weighted sum of two contributions: the first summation counts the number of installed transceiver pairs, the second summation computes the sum of the spectrum slices occupied over all network links. By properly tuning the value of the multiplicative factors $\alpha_{1}, \alpha_{2}$, either transceivers minimization (for $\alpha_{1} \gg \alpha_{2}$ ) or spectrum minimization (for $\alpha_{1} \ll \alpha_{2}$ ) can be privileged.

Constraints (2) state that each traffic demand is served exactly by one channel along one lightpath; constraints (3) state that each traffic demand uses only one modulation format; constraints (4) state that traffic demand $t$ must be satisfied by the total capacity of the transceivers installed at the two end nodes; constraints (5) state that modulation format $m$ can be used on lightpath $k$ if its reach exceeds the lightpath length and if lightpath $k$ is chosen to route a traffic request; constraints (6) imposes that all the transceivers installed on lightpath $k$ use the same modulation format $m$; constraints (7) ensure that the width of channel $c$ used to serve a traffic request is sufficiently large to accommodate all the required transceivers and the guardband $G$; constraints (8) state that two spectrally overlapping channels cannot be activated simultaneously along lightpaths that share at least one link.

In the so called "reach" constraints (5), we observe that the binary parameter $\lambda_{k}^{m}$ encodes the information on whether the expected BER is below threshold th for a lightpath $k$ using modulation format $m$. In this paper we compare two possible ways of computing such parameter:

- in the margined approach, we compute $\lambda_{k}^{m}$ with the classical margined formulas where the reach (i.e. maximum distance) coverable for a given modulation format and traffic volume is precomputed as in [5].
- in the ML-based and iterative approaches, we compute $\lambda_{k}^{m}$ exploiting the output of the ML classifier described in subsection III-A.

In particular, given a reference value $p_{t h}^{*}, \lambda_{k}^{m}$ is set as follows:

$$
\lambda_{k}^{m}=\left\{\begin{array}{cc}
1 & \text { if } P\left(\operatorname{BER}\left(\mathcal{L}_{k}, \mathcal{L}_{k}^{\max }, l_{k}, d_{t}, m\right) \leq t h\right) \geq p_{t h}^{*} \\
0 & \text { otherwise }
\end{array}\right.
$$

Since we aim at reducing the number of false positives at the price of a higher number of false negatives, in Section VI we explore different values of $p_{t h}^{*}$ in the range [0.5, 0.99].

\section{Additional Constraints for the Iterative Design Approach}

Equations (1)-(8) compute an optimal RMSA solution without taking into account potential interferences among lightpaths deployed on adjacent channels and their effects on reach constraints. For a lightpath $k$ serving traffic request $t=(s, d)$ with modulation format $m$ belonging to the optimal solution and exhibiting near to threshold BER, the risk exists that the estimated $p_{t h}$ is affected by an error, which may in turn impact on the value of parameter $\lambda_{k}^{m}$. To reduce such error, the characteristics of neighbor lightpaths should be included as classification features: in particular, according to [10], the traffic volumes $d_{l}$ and $d_{r}$ served by the two lightpaths $k_{l}$ and $k_{r}$ co-propagating with $k$ on at least one link using modulation formats $m_{l}$ and $m_{r}$ and deployed on the spectrally nearest left and right channels $c_{l}$ and $c_{r}$ with guardband widths $g_{l}$ and $g_{r}$ should be considered ${ }^{6}$.

To capture the impact of inter-channel crosstalk while limiting the explosion in computational complexity, we adopt the iterative design approach. At iteration $i t$, given an optimal solution $s_{i t}^{*}=\left(b_{i t}^{*}, \beta_{i t}^{*}, y_{i t}^{*}\right)$ to (1)-(8), for each lightpath belonging to $s_{i t}^{*}$ we query the ML classifier using the additional features describing its adjacent deployed lightpaths. If for at least one of the lightpaths belonging to $s_{i t}^{*}$ the classifier returns as output a probability $p_{t h}$ lower than $p_{t h}^{*}$, then we add new constraints to (1)-(8) in order to cut off $s^{*}$ from the feasible solution set and we iterate by solving the new problem again. More in detail, to eliminate $s^{*}$ we add a pair of constraints, called modulation cuts, for each deployed lightpath $k$ belonging to $s^{*}$ that exhibits a BER lower than th with probability $p_{t h} \leq p_{t h}^{*}$. Given the unfeasible triplet deployed lightpaths $\left(k, k_{l}, k_{r}\right)$ and their respective modulation formats $\left(m, m_{l}, m_{r}\right)$, modulation cuts read as follows:

$$
\begin{aligned}
& \beta_{k}^{m}+\beta_{k_{l}}^{m_{l}} \leq 1 \\
& \beta_{k}^{m}+\beta_{k_{r}}^{m_{r}} \leq 1
\end{aligned}
$$

The iterative approach stops when the probability $p_{t h}$ that the estimated BER is below the target threshold $t h$ is higher than the reference value $p_{t h}^{*}$ for all deployed lightpaths or when a stopping criterion, either on computational time or number of iterations, is reached.

Note that an alternative brute-force approach would be the precomputation of $p_{t h}$ for every possible combination of

\footnotetext{
${ }^{6}$ Note that $g_{l}$ and $g_{r}$ can be greater than $G$ due to potential spectrum fragmentation.
} 
the values of $d_{l}, d_{r}, g_{l}, g_{m}, m_{l}$ and $m_{r}$. As the number of such parameters grows as $O\left(|M|^{3} \cdot|K|^{3} \cdot|D|^{3} \cdot|S|^{2}\right)$, where $S$ is the set of spectrum slices, a brute-force approach is computationally unmanageable in realistic network scenarios.

\section{NUMERICAL RESULTS}

\section{A. Simulation Framework}

We consider the Japan network topology depicted in Fig. 3 (with 14 nodes and 22 links). We assume the usage of a flexible grid with standard slice width of $F=12.5 \mathrm{GHz}$ [4] and elastic transceivers with optical bandwidth $B=$ $37.5 \mathrm{GHz}$ (i.e., 3 slices). The guardband size is set to $G=$ $F=12.5 \mathrm{GHz}$. The available spectrum over each link is $4 \mathrm{THz}$ (i.e., 320 slices). The set $M$ of modulation formats includes dual polarization (DP)-BPSK, DP-QPSK and DP$n$-QAM, with $n=8 ; 16 ; 32 ; 64$, resulting in capacities of $50,100,150,200,250,300 \mathrm{Gbps}$, respectively. We consider 5 all-to-all traffic matrices (i.e., $|T|=14 \times 13=182$ ) with random traffic requests uniformly distributed among the sampling set $\mathcal{M}=\{50,100,150,200,250,300,350,400\}$ Gbps. It follows that, in our instances, superchannels consisting of up to 8 adjacent transceivers can be deployed (e.g., in the case of a 400 Gbps request served with DP-BPSK). Margined transmission reaches for each combination of traffic volume and modulation format are reported in Table I.

Ten considered problem instances are listed in Table II, including their total traffic, maximum and average traffic per request, and number of candidate shortest paths $\bar{k}$ per request. For instances with $\bar{k}=1$ we consider 5 different sets of modulation formats, where the most spectrally efficient format ranges from QPSK to 64-QAM (it follows that the first set comprises only BPSK and QPSK, whereas the last set ranges from BPSK to 64-QAM), while for instances with $\bar{k}=2$ we tested only the full range of available modulation formats (i.e., from BPSK to 64-QAM). The instances we consider are representative for nation-wide networks where link distances are in order of hundreds of kilometers (e.g., from 40 to 320 in the Japan case). For such networks the traffic we consider ranges from low congested cases, with average traffic of 146.7 Gbps per link to high congested cases of 264.0 Gbps per link. Note that traffic requests are generated so that, for the corresponding shortest path, there exists at least one feasible modulation format able to carry the traffic considering the margined reach values. In other words, for long distance pairs, large traffic volumes may be excluded from set $\mathcal{M}$ (as an example, for traffic generated by node 1 and destined to node 13 , the shortest path is $1120 \mathrm{~km}$ long, which imposes that at most $150 \mathrm{Gbps}$ can be transmitted using BPSK).

In the ILP objective function we set $\alpha_{1} \ll \alpha_{2}$, i.e., we privilege spectrum minimization, but, if multiple optimal solutions exist, the one minimizing the total number of installed transceiver is selected.

To verify the feasibility of the found solutions, we generate the actual received BER of the various lightpaths in the optimal solution using Qtool described in Section III-B.
TABLE I: Margined reaches expressed in $\mathrm{km}$ for a given traffic demand and modulation format (computed as in [5])

\begin{tabular}{rrrrrrrrr}
\hline & \multicolumn{8}{c}{ traffic volume [Gbps] } \\
& 50 & 100 & 150 & 200 & 250 & 300 & 350 & 400 \\
\hline BPSK & 3400 & 1700 & 1200 & 900 & 700 & 600 & 500 & 400 \\
QPSK & 3300 & 1700 & 1100 & 900 & 700 & 600 & 500 & 400 \\
8-QAM & 1300 & 700 & 400 & 300 & 300 & 200 & 200 & 100 \\
16-QAM & 1000 & 500 & 300 & 200 & 200 & 200 & 100 & 100 \\
32-QAM & 500 & 200 & 100 & 100 & 100 & 100 & 0 & 0 \\
64-QAM & 300 & 100 & 100 & 100 & 0 & 0 & 0 & 0 \\
\hline
\end{tabular}

TABLE II: Instances of the problem

\begin{tabular}{rrrrr}
\hline Instance & Total [Tbps] & Max [Gbps] & Avg [Gbps] & $\bar{k}$ \\
\hline 1 & 26.70 & 200 & 146.70 & 1 \\
2 & 33.75 & 300 & 185.44 & 1 \\
3 & 38.45 & 400 & 211.26 & 1 \\
4 & 42.90 & 300 & 235.71 & 1 \\
5 & 48.05 & 400 & 264.01 & 1 \\
6 & 26.70 & 200 & 146.70 & 2 \\
7 & 33.75 & 300 & 185.44 & 2 \\
8 & 38.45 & 400 & 211.26 & 2 \\
9 & 42.90 & 300 & 235.71 & 2 \\
10 & 48.05 & 400 & 264.01 & 2 \\
\hline
\end{tabular}

For the ML-based and iterative approaches, the two classifiers have been trained with 20000 randomly sampled instances $^{7}$. For classifier 1 , the instances included the first five features reported in Fig. 1, whereas for classifier 2, all the 11 features reported in Fig. 1 were used. In the following, we denote the probabilistic outputs of the two classifiers as $p_{t h}^{1}$ and $p_{t h}^{2}$, respectively. We explore different values of $p_{t h}^{*}$ in the set $\{0.5,0.75,0.90,0.99\}$.

\section{B. Results with Margined Design Approach}

We first solve the ILP formulation with the margined approach for all instances in Table II. These results will be used as benchmark to calculate the spectrum-occupation reduction achieved by the ML-based and iterative approaches. In Fig. 4, we include results for all the feasible instances ${ }^{8}$. For the optimal RMSA solution of each instance and modulation format in use, we report the overall spectrum occupation and the highest BER of any of the deployed lightpaths.

We note that: $i$ ) the margined approach does not admit any solution for instances $3,4,5$ and $8,9,10$. Since in our problem instances all traffic demands admit at least one feasible modulation format to be used for transmission, the infeasibility is due to an insufficient link capacity for instances with total traffic larger or equal to $38.45 \mathrm{Tbps}$; $i$ ) in all solutions, no ligthpath violates the BER threshold th (BER always remains at least two orders of magnitude below th), confirming that the margined approach is very conservative and guarantees lightpath feasibility.

\footnotetext{
${ }^{7}$ Note that, as in this paper we focus on the integration of the QoT classification outputs in RMSA, we assume that the classifier is well-trained and leave the study on the impact of incomplete or biased training datasets for future work.

${ }^{8}$ Note that the legend on the $\mathrm{x}$-axis shall be read as the highest modulation format in a certain instance (e.g., 32-QAM-1 refers to the variation of instance with $\bar{k}=1$ that considers modulation formats from BPSK to 32-QAM
} 


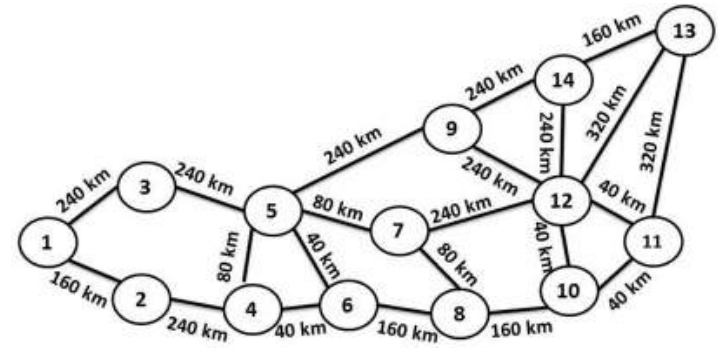

Fig. 3: Japan network topology

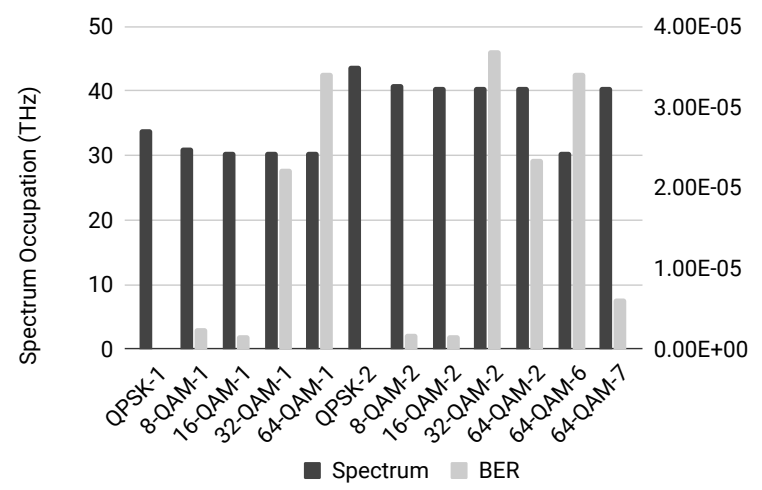

Fig. 4: Spectrum occupancy and BER with Margined Reaches. Missing BER columns indicate BER values below $10^{-8}$.

\section{Results with ML-Based Design Approach}

Figure 5 reports results of the ML-based approach, for those instances that could also be solved with the margined approach (i.e., instances 1, 2, 6 and 7). In Fig. 5a, on the left axis we report the spectrum occupancy in $\mathrm{THz}$ and on the right axis we report the percentage savings of ML-Based approach with respect to the margined approach. Columns are stacked as both spectrum occupancy and savings are monotonically decreasing (resp. increasing) lowering the value of $p_{t h}^{*}$. Figure $5 \mathrm{~b}$ reports the worst obtained BER (among all lightpaths) and the number of ligthpaths in the optimal solution for which the BER calculated with the Qtool is above threshold. We observe remarkable savings, up to $36 \%$. The ML-based approach is less conservative that the margined approach hence it allows to use more spectrally-efficient modulation formats. We observe larger savings when the reference probability value is lower (i.e. $p_{t h}^{*}=0.5$ ), though in these cases the BER is sometimes not acceptable: for example, in instance 7 using modulation formats up to 64-QAM, for 5 lightpaths out of 182 the value is larger than $t h^{9}$. For higher probability reference values (i.e. $p_{t h}^{*} \in\{0.75,0.9\}$ ) the BER is most of the time below the threshold. In few cases it is larger, but the violation is marginal. More in detail, when the set of available

\footnotetext{
${ }^{9}$ There are two possible causes for such behavior: first, the value of $p_{t h}^{1}$ output by the classifier may not exactly match the true probability (despite accuracy values up to $98 \%$ reported in [10, Section VI], prediction errors may occur). Secondly, the Qtool estimation process includes the addition of an exponentially distributed random transmission penalty [10]: therefore, on input of the same set of parameters, multiple estimations obtained with the Qtool will slightly differ in their value, which could make the difference in the evaluation of near-to-threshold BERs.
}

TABLE III: BER with ML based approach

\begin{tabular}{|c|c|c|c|c|c|c|c|}
\hline Mod. & $p_{t h}^{*}$ & W. BER & $\#$ & W. BER & $\#$ & W. BER & $\#$ \\
\hline & & \multicolumn{2}{|c|}{ Instance 3} & \multicolumn{2}{|c|}{ Instance 4} & \multicolumn{2}{|c|}{ Instance 5} \\
\hline QPSK & 0.5 & 4.34E-09 & 0 & $6.65 \mathrm{E}-09$ & 0 & 4.34E-09 & 0 \\
\hline QPSK & 0.75 & $4.18 \mathrm{E}-09$ & 0 & $6.65 \mathrm{E}-09$ & 0 & $4.18 \mathrm{E}-09$ & 0 \\
\hline QPSK & 0.9 & 4.34E-09 & 0 & $6.65 \mathrm{E}-09$ & 0 & $4.18 \mathrm{E}-09$ & 0 \\
\hline QPSK & 0.99 & $4.18 \mathrm{E}-09$ & 0 & $9.01 \mathrm{E}-09$ & 0 & $9.01 \mathrm{E}-09$ & 0 \\
\hline 8-QAM & 0.5 & $4.61 \mathrm{E}-03$ & 2 & $1.40 \mathrm{E}-02$ & 5 & $1.94 \mathrm{E}-02$ & 5 \\
\hline 8-QAM & 0.75 & $4.64 \mathrm{E}-03$ & 1 & $1.35 \mathrm{E}-02$ & 5 & $1.14 \mathrm{E}-02$ & 3 \\
\hline 8-QAM & 0.9 & $3.16 \mathrm{E}-03$ & 0 & $2.45 \mathrm{E}-03$ & 0 & $3.16 \mathrm{E}-03$ & 0 \\
\hline 8-QAM & 0.99 & $1.02 \mathrm{E}-03$ & 0 & $1.36 \mathrm{E}-03$ & 0 & $8.04 \mathrm{E}-04$ & 0 \\
\hline 16-QAM & 0.5 & $1.07 \mathrm{E}-02$ & 3 & $1.82 \mathrm{E}-02$ & 8 & $1.27 \mathrm{E}-02$ & 7 \\
\hline 16-QAM & 0.75 & $1.33 \mathrm{E}-02$ & 1 & $1.96 \mathrm{E}-02$ & 8 & $4.64 \mathrm{E}-03$ & 4 \\
\hline 16-QAM & 0.9 & $1.41 \mathrm{E}-03$ & 0 & $3.88 \mathrm{E}-03$ & 0 & $2.16 \mathrm{E}-03$ & 0 \\
\hline 16-QAM & 0.99 & $8.13 \mathrm{E}-04$ & 0 & 8.33E-04 & 0 & $8.13 \mathrm{E}-04$ & 0 \\
\hline 32-QAM & 0.5 & $1.37 \mathrm{E}-02$ & 5 & $1.95 \mathrm{E}-02$ & 9 & $1.54 \mathrm{E}-02$ & 15 \\
\hline 32-QAM & 0.75 & $1.34 \mathrm{E}-02$ & 3 & $1.31 \mathrm{E}-02$ & 5 & $1.25 \mathrm{E}-02$ & 6 \\
\hline 32-QAM & 0.9 & $6.75 \mathrm{E}-03$ & 1 & $5.67 \mathrm{E}-03$ & 2 & $3.65 \mathrm{E}-03$ & 0 \\
\hline 32-QAM & 0.99 & $2.98 \mathrm{E}-03$ & 0 & $1.54 \mathrm{E}-03$ & 0 & $9.38 \mathrm{E}-04$ & 0 \\
\hline 64-QAM & 0.5 & $1.96 \mathrm{E}-02$ & 3 & $1.25 \mathrm{E}-02$ & 10 & $2.38 \mathrm{E}-02$ & 20 \\
\hline 64-QAM & 0.75 & $6.40 \mathrm{E}-03$ & 2 & $1.24 \mathrm{E}-02$ & 7 & $2.06 \mathrm{E}-02$ & 7 \\
\hline 64-QAM & 0.9 & $6.11 \mathrm{E}-03$ & 1 & $4.88 \mathrm{E}-03$ & 1 & $1.58 \mathrm{E}-02$ & 3 \\
\hline \multirow[t]{2}{*}{ 64-QAM } & 0.99 & $4.00 \mathrm{E}-03$ & 0 & $1.25 \mathrm{E}-03$ & 0 & $9.20 \mathrm{E}-03$ & 2 \\
\hline & & \multicolumn{2}{|c|}{ Instance 8} & \multicolumn{2}{|c|}{ Instance 9} & \multicolumn{2}{|c|}{ Instance 10} \\
\hline 64-QAM & 0.5 & $1.85 \mathrm{E}-02$ & 6 & $1.56 \mathrm{E}-02$ & 15 & $2.42 \mathrm{E}-02$ & 19 \\
\hline 64-QAM & 0.75 & $9.32 \mathrm{E}-03$ & 3 & $8.90 \mathrm{E}-03$ & 4 & $1.27 \mathrm{E}-02$ & 5 \\
\hline 64-QAM & 0.9 & $2.98 \mathrm{E}-03$ & 0 & $4.91 \mathrm{E}-03$ & 2 & $3.53 \mathrm{E}-03$ & 0 \\
\hline 64-QAM & 0.99 & $3.50 \mathrm{E}-03$ & 0 & $3.10 \mathrm{E}-03$ & 0 & $3.53 \mathrm{E}-03$ & 0 \\
\hline
\end{tabular}

modulation formats is limited to BPSK and QPSK, savings up to $11 \%$ can be obtained without any violation of th (the highest BER values remain below $10^{-8}$ ). When 8 -QAM is introduced, savings raise up to almost $27 \%$, with only one lightpath experiencing above-threshold BER $\left(5.51 \cdot 10^{-3}\right.$ vs. a threshold of $4 \cdot 10^{-3}$ in instance 2) among all the considered instances. Using more spectrally-efficient modulation formats up to 64-QAM further increases savings up to $36 \%$, at the price of a few lightpaths exhibiting over-threshold BERs. For those lightpaths, the highest experienced BER never raises above $10^{-2}$. Finally, for very conservative scenarios, i.e. when $p_{t h}^{*}=0.99$, the obtained BERs are always below th. Even in these cases, savings up to $27 \%$ can be obtained when the whole range of modulation formats up to 64-QAM is adopted.

Table III reports results, related to the ML-based approach, for those instances that could not be solved with the marginedreach approach. Note that the ML-based approach is able to provide solutions to all instances (while the margined approach failed for instances $3,4,5,8,9$, and 10). We now report BER performances only. Due to space restrictions, results are reported in two columns and column headers are compacted. For $p_{t h}^{*}=0.99$, we observe BER values always below th for all instances, with the only exception of instance 5 (i.e. with large traffic demand and $\bar{k}=1$ ): in that case, when the modulation format ranges up to 64-QAM, two lightpaths exhibit too high BER. Note that, since only one candidate lightpath per node pair is considered, in this scenario the problem reduces to modulation format and spectrum assignment, whereas the routing is predefined. Interestingly, for instance 10 (which uses the same demand matrix but with $\bar{k}=2$, i.e., the demand can 


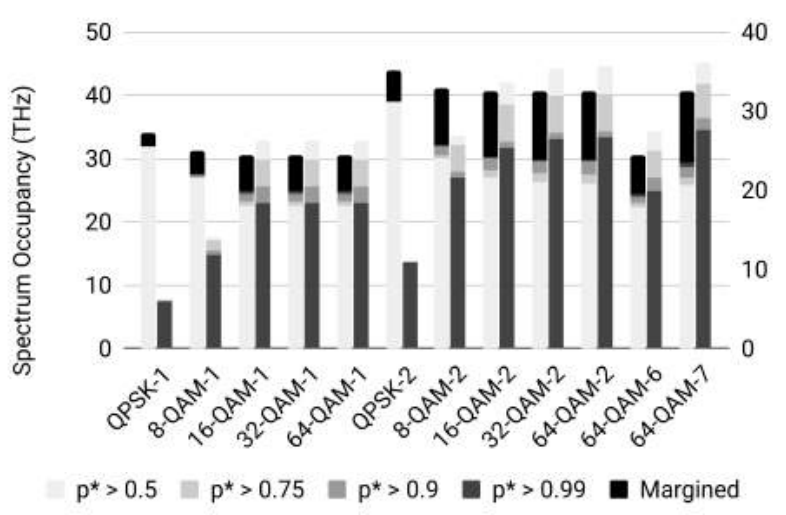

(a) Spectrum Occupation

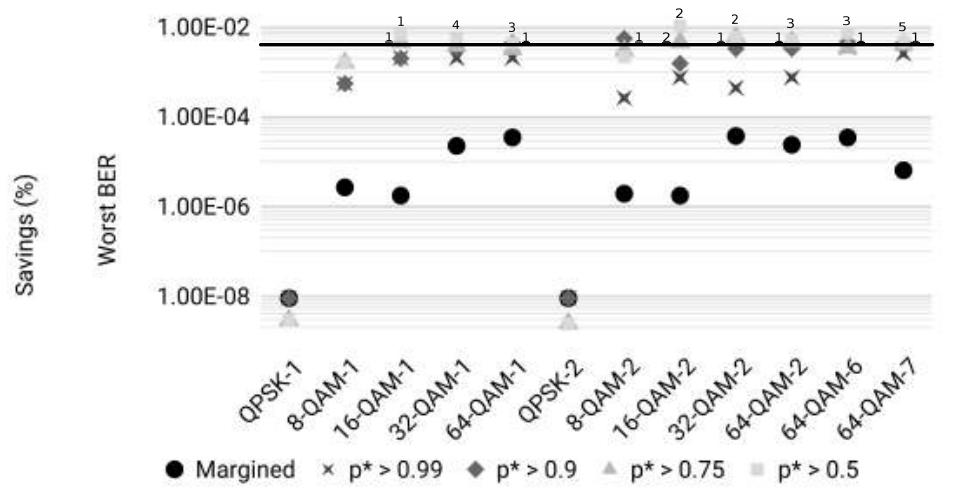

(b) Markers indicate the Worst BER. Numbers above square, left to triangular and right to diamond markers indicate the corresponding amount of above-threshold lightpaths

Fig. 5: Comparison of Margined vs. ML-Based approaches

be routed over two different paths), no threshold violation is observed. The increased flexibility introduced by having two candidate lightpaths per node pair is therefore beneficial.

\section{Results with Iterative Approach}

Table IV reports results obtained with the iterative approach. Due to space limitations, We restrict our analysis to instances with modulation formats up to 64-QAM. The column labeled with "It." reports the number of iterations before reaching the final solution. Results marked with an asterisk " $*$ " denote the fact that, after the reported number of iterations, the additional cuts render the problem infeasible. In such cases, the last feasible solution is reported. Columns labeled with " $|M C|$ " report the number of added modulation cuts. The last two columns report the percentage increase in the objective function with respect to the solution obtained before addition of any modulation cut (i.e., at the first iteration round) and the savings with respect to the margined approach (note that, when savings are not reported, it means that the margined approach found no feasible solution). Remarkably, when comparing the results to those in Table III, we observe that for $p_{t h}^{*} \in\{0.9,0.99\}$ the iterative approach is able to provide a solution with BER below th for all but only two instances (i.e., instances 1 and 7 with $p_{t h}^{*}=0.9$, exhibiting one above-threshold lightpath each), which was not achievable without using the modulation cuts. Moreover, for $p_{t h}^{*} \in\{0.5,0.75\}$, the number of lightpaths with above-threshold BER is largely reduced with respect to Table III. This comes at the price of a small reduction of the spectrum savings, as some profitable but risky solutions are eliminated by modulation cuts. Nevertheless, savings above $30 \%$ are obtained, showing that the iterative approach achieves significant reductions in spectrum occupation in comparison to the margined approach, while almost completely eliminating deployed lightpaths with above-threshold BER.

\section{E. Complexity Discussion}

In the ILP formulation presented in section $\mathrm{V}$, the number of variables grows as $O(|K| \cdot(|M|+|C|))$, whereas the number
TABLE IV: BER with iterative approach

\begin{tabular}{|c|c|c|c|c|c|c|c|}
\hline In. & $p_{t h}^{*}$ & It. & $|M C|$ & W. Ber & \# & Add. C. $\%$ & Sav. $\%$ \\
\hline 1 & 0.5 & 18 & 134 & $6.47 \mathrm{E}-03$ & 1 & $4.45 \%$ & $22.89 \%$ \\
\hline 1 & 0.75 & 24 & 348 & $1.35 \mathrm{E}-03$ & 0 & $8.07 \%$ & $17.17 \%$ \\
\hline 1 & 0.9 & $16 *$ & 826 & $4.11 \mathrm{E}-03$ & 1 & $22.10 \%$ & $-2.21 \%$ \\
\hline 1 & 0.99 & $6 *$ & 1104 & $2.98 \mathrm{E}-03$ & 0 & $20.68 \%$ & $-2.81 \%$ \\
\hline 2 & 0.5 & 8 & 68 & $5.76 \mathrm{E}-03$ & 2 & $2.40 \%$ & $34.13 \%$ \\
\hline 2 & 0.75 & 23 & 236 & $1.18 \mathrm{E}-03$ & 0 & $7.83 \%$ & $26.30 \%$ \\
\hline 2 & 0.9 & $13 *$ & 670 & $3.92 \mathrm{E}-03$ & 0 & $18.11 \%$ & $11.29 \%$ \\
\hline 2 & 0.99 & $5 *$ & 870 & $1.67 \mathrm{E}-03$ & 0 & $21.86 \%$ & $6.08 \%$ \\
\hline 3 & 0.5 & 2 & 16 & $5.43 \mathrm{E}-03$ & 1 & $-0.40 \%$ & - \\
\hline 3 & 0.75 & 30 & 260 & $1.67 \mathrm{E}-03$ & 0 & $7.29 \%$ & - \\
\hline 3 & 0.9 & $13 *$ & 840 & $2.77 \mathrm{E}-03$ & 0 & $20.91 \%$ & - \\
\hline 3 & 0.99 & $6 *$ & 1148 & $2.79 \mathrm{E}-03$ & 0 & $24.37 \%$ & - \\
\hline 4 & 0.5 & 18 & 154 & $6.70 \mathrm{E}-03$ & 2 & $5.66 \%$ & - \\
\hline 4 & 0.75 & 7 & 132 & $2.03 \mathrm{E}-03$ & 0 & $5.36 \%$ & - \\
\hline 4 & 0.9 & $10 *$ & 614 & $4.37 \mathrm{E}-04$ & 0 & $21.94 \%$ & - \\
\hline 4 & 0.99 & $5 *$ & 938 & $1.52 \mathrm{E}-03$ & 0 & $27.81 \%$ & - \\
\hline 5 & 0.5 & 17 & 114 & $5.21 \mathrm{E}-03$ & 2 & $3.21 \%$ & - \\
\hline 5 & 0.75 & 13 & 164 & $9.62 \mathrm{E}-04$ & 0 & $6.13 \%$ & - \\
\hline 5 & 0.9 & $11 *$ & 674 & $2.10 \mathrm{E}-03$ & 0 & $23.51 \%$ & - \\
\hline 5 & 0.99 & $1 *$ & 260 & $2.10 \mathrm{E}-03$ & 0 & $-0.96 \%$ & - \\
\hline 6 & 0.5 & 30 & 186 & $9.01 \mathrm{E}-09$ & 0 & $1.80 \%$ & $26.06 \%$ \\
\hline 6 & 0.75 & 30 & 714 & $4.18 \mathrm{E}-03$ & 1 & $7.56 \%$ & $18.90 \%$ \\
\hline 6 & 0.9 & 30 & 1744 & $1.77 \mathrm{E}-03$ & 0 & $15.41 \%$ & $7.31 \%$ \\
\hline 6 & 0.99 & $7 *$ & 1370 & $3.00 \mathrm{E}-03$ & 0 & $12.26 \%$ & $8.75 \%$ \\
\hline 7 & 0.5 & 17 & 116 & $1.67 \mathrm{E}-03$ & 0 & $1.33 \%$ & $35.21 \%$ \\
\hline 7 & 0.75 & 30 & 646 & $6.21 \mathrm{E}-03$ & 1 & $8.85 \%$ & $26.97 \%$ \\
\hline 7 & 0.9 & $11 *$ & 636 & $2.56 \mathrm{E}-03$ & 0 & $9.50 \%$ & $21.70 \%$ \\
\hline 7 & 0.99 & $5 *$ & 938 & $1.32 \mathrm{E}-03$ & 0 & $11.44 \%$ & $18.23 \%$ \\
\hline 8 & 0.5 & 28 & 172 & $6.73 \mathrm{E}-04$ & 0 & $2.33 \%$ & - \\
\hline 8 & 0.75 & 30 & 572 & $3.81 \mathrm{E}-03$ & 0 & $7.79 \%$ & - \\
\hline 8 & 0.9 & $12 *$ & 732 & $4.55 \mathrm{E}-03$ & 1 & $10.65 \%$ & - \\
\hline 8 & 0.99 & $5 *$ & 990 & 3.93E-03 & 0 & $13.24 \%$ & - \\
\hline 9 & 0.5 & 23 & 172 & $1.24 \mathrm{E}-02$ & 2 & $3.41 \%$ & - \\
\hline 9 & 0.75 & 30 & 468 & $4.53 \mathrm{E}-03$ & 1 & $8.56 \%$ & - \\
\hline 9 & 0.9 & $10 *$ & 632 & $1.67 \mathrm{E}-03$ & 0 & $10.54 \%$ & - \\
\hline 9 & 0.99 & $5 *$ & 900 & $1.32 \mathrm{E}-03$ & 0 & $13.14 \%$ & - \\
\hline 10 & 0.5 & $6 *$ & 90 & $1.64 \mathrm{E}-02$ & 12 & $1.81 \%$ & - \\
\hline 10 & 0.75 & $15 *$ & 338 & $1.63 \mathrm{E}-02$ & 1 & $6.54 \%$ & - \\
\hline 10 & 0.9 & $8 *$ & 528 & $3.83 \mathrm{E}-03$ & 0 & $9.33 \%$ & - \\
\hline 10 & 0.99 & $4 *$ & 812 & $9.20 \mathrm{E}-04$ & 0 & $12.18 \%$ & - \\
\hline
\end{tabular}

of constraints in Eqs.(2)-(7) grows as $O(|T|+|M| \cdot|K|)$. Conversely, the number of constraints in Eq. (8) strictly depends on the definition of the channel set $C$ and on the sparsity of the network and the size of the path set $K$. As 
the number of these constraints can grow very rapidly, in the order of $O\left(|C|^{2} \cdot|K|^{2}\right)$ in the worst case, some care must be adopted in designing set $C$ to limit its cardinality, e.g., by considering only channels of sizes $n B+G$, with $n$ integer. In our experiments, the average number of variables for an ILP problem for the ML-based approach is 67815.1, the average number of constraints is 68984.4 , while the coefficient matrix has about 1 million non-zero coefficients. The ILP is solved by Gurobi [23]. Each iteration requires, in average, 26.4 seconds for instances 1-5 and 53.6 seconds for instances 6-10 on a $3.2 \mathrm{GHz}$ processor with 4 cores and $32 \mathrm{~GB}$ of RAM, Linux operating system. According to these results, we remark that the proposed approach may not scale well for larger networks as computational complexity grows non linearly with network size. Therefore, a heuristic approach to solve larger instances will be topic of future investigation.

\section{CONCLUSION}

We propose a network planning framework that integrates the probabilistic outputs of a ML-based QoT estimator for candidate optical lightpaths in an ILP for routing and spectrum assignment, considering multiple modulation formats. Results show spectrum savings up to $30 \%$ with respect to traditional approaches based on margined reaches. By properly tuning the minimum value of the probability of satisfying a given bit error rate threshold (returned by the classifier and required to consider a candidate lightpath as acceptable), network configurations characterized by different risk levels - which are quantified by the number of lightpaths that exceed a given bit error rate system threshold - can be obtained. The number of "risky" lightpaths can be further reduced if an iterative procedure is adopted, in which supplementary constraints are added to the linear program based on the outputs of additional queries issued to the classifier, which include as features some characteristics of the neighbor channels.

\section{ACKNOLEDGEMENTS}

Massimo Tornatore acknowledges the support of the European Community under grant agreement no. 761727 MetroHaul project, and the support of Science Foundation grant 1716945.

\section{REFERENCES}

[1] Cisco, "Cisco Visual Networking Index: Global Mobile Data Traffic Forecast Update, 20152020,” Feb. 2016.

[2] O. Gerstel, M. Jinno, A. Lord, and S. B. Yoo, "Elastic optical networking: A new dawn for the optical layer?" IEEE Communications Magazine, vol. 50, no. 2, 2012.

[3] S. Talebi, F. Alam, I. Katib, M. Khamis, R. Salama, and G. N. Rouskas, "Spectrum management techniques for elastic optical networks: A survey," Optical Switching and Networking, vol. 13, pp. 34-48, 2014.

[4] International Telecommunication Union, Telecommunication Standardization Sector, "Spectral grids for WDM applications: DWDM frequency grid," ITU-T Rec. G.694.1, Feb. 2012. [Online]. Available: http://www.itu.int

[5] G. Bosco, V. Curri, A. Carena, P. Poggiolini, and F. Forghieri, "On the performance of nyquist-WDM terabit superchannels based on PMBPSK, PM-QPSK, PM-8QAM or PM-16QAM subcarriers,' IEEE/OSA Journal of Lightwave Technology, vol. 29, no. 1, pp. 53-61, 2011.
[6] R. Proietti, X. Chen, A. Castro, G. Liu, H. Lu, K. Zhang, J. Guo, Z. Zhu, L. Velasco, and S. J. B. Yoo, "Experimental demonstration of cognitive provisioning and alien wavelength monitoring in multi-domain eon," in Optical Fiber Communication Conference. Optical Society of America, 2018, p. W4F.7. [Online]. Available: http://www.osapublishing.org/abstract.cfm?URI=OFC-2018-W4F.7

[7] Y. Pointurier, "Design of low-margin optical networks," IEEE/OSA Journal of Optical Communications and Networking, vol. 9, no. 1, pp. A9-A17, 2017.

[8] E. Ip and J. M. Kahn, "Compensation of dispersion and nonlinear impairments using digital backpropagation," Journal of Lightwave Technology, vol. 26, no. 20, pp. 3416-3425, Oct 2008.

[9] K. Christodoulopoulos, P. Kokkinos, A. Di Giglio, A. Pagano, N. Argyris, C. Spatharakis, S. Dris, H. Avramopoulos, J. Antona, C. Delezoide et al., "Orchestra-optical performance monitoring enabling flexible networking," in Transparent Optical Networks (ICTON), 2015 17th International Conference on. IEEE, 2015, pp. 1-4.

[10] C. Rottondi, L. Barletta, A. Giusti, and M. Tornatore, "Machine-learning method for quality of transmission prediction of unestablished lightpaths," IEEE/OSA Journal of Optical Communications and Networking, vol. 10, no. 2, pp. A286-A297, Feb 2018.

[11] T. Jiménez, J. C. Aguado, I. de Miguel, R. J. Durán, M. Angelou, N. Merayo, P. Fernández, R. M. Lorenzo, I. Tomkos, and E. J. Abril, "A cognitive quality of transmission estimator for core optical networks," IEEE/OSA Journal of Lightwave Technology, vol. 31, no. 6, pp. 942951, Jan. 2013.

[12] E. Seve, J. Pesic, C. Delezoide, and Y. Pointurier, "Learning process for reducing uncertainties on network parameters and design margins," in Optical Fiber Communications Conference (OFC) 2017. IEEE, Mar. 2017, pp. 1-3.

[13] J. Thrane, J. Wass, M. Piels, J. C. M. Diniz, R. Jones, and D. Zibar, "Machine Learning Techniques for Optical Performance Monitoring From Directly Detected PDM-QAM Signals,' IEEE/OSA Journal of Lightwave Technology, vol. 35, no. 4, pp. 868-875, Feb. 2017.

[14] Y. Pointurier, M. Coates, and M. Rabbat, "Cross-layer monitoring in transparent optical networks," IEEE/OSA Journal of Optical Communications and Networking, vol. 3, no. 3, pp. 189-198, Feb. 2011.

[15] N. Sambo, Y. Pointurier, F. Cugini, L. Valcarenghi, P. Castoldi, and I. Tomkos, "Lightpath establishment assisted by offline QoT estimation in transparent optical networks," IEEE/OSA Journal of Optical Communications and Networking, vol. 2, no. 11, pp. 928-937, Mar. 2010.

[16] M. Angelou, Y. Pointurier, D. Careglio, S. Spadaro, and I. Tomkos, "Optimized monitor placement for accurate QoT assessment in core optical networks," IEEE/OSA Journal of Optical Communications and Networking, vol. 4, no. 1, pp. 15-24, Dec. 2012.

[17] I. Sartzetakis, K. Christodoulopoulos, C. Tsekrekos, D. Syvridis, and E. Varvarigos, "Quality of transmission estimation in WDM and elastic optical networks accounting for space-spectrum dependencies," IEEE/OSA Journal of Optical Communications and Networking, vol. 8, no. 9, pp. 676-688, Sep. 2016.

[18] B. C. Chatterjee, N. Sarma, and E. Oki, "Routing and spectrum allocation in elastic optical networks: A tutorial," IEEE Communications Surveys \& Tutorials, vol. 17, no. 3, pp. 1776-1800, 2015.

[19] T. Panayiotou, S. Chatzis, and G. Ellinas, "Performance analysis of a data-driven quality-of-transmission decision approach on a dynamic multicast-capable metro optical network," IEEE/OSA Journal of Optical Communications and Networking, vol. 9, no. 1, pp. 98-108, Jan. 2017.

[20] Q. Yao, H. Yang, R. Zhu, A. Yu, W. Bai, Y. Tan, J. Zhang, and H. Xiao, "Core, mode, and spectrum assignment based on machine learning in space division multiplexing elastic optical networks," IEEE ACCESS, vol. 6, pp. $15898-15907,2018$.

[21] X. Chen, R. Proietti, H. Lu, A. Castro, and S. B. Yoo, "Knowledgebased autonomous service provisioning in multi-domain elastic optical networks," IEEE Communications Magazine, vol. 56, no. 8, pp. 152$158,2018$.

[22] M. Tornatore, C. Rottondi, R. Goscien, K. Walkowiak, G. Rizzelli, and A. Morea, "On the complexity of routing and spectrum assignment in flexible-grid ring networks," IEEE/OSA Journal of Optical Communications and Networking, vol. 7, no. 2, pp. A256-A267, 2015.

[23] L. Gurobi Optimization, "Gurobi optimizer reference manual," 2018. [Online]. Available: http://www.gurobi.com 